\title{
The Influence of Culture on Analgesic Consumption: A Retrospective Study among Malaysian Patients after Breast Cancer Surgery
}

\author{
Salimah Japar1 ${ }^{*}$, Soh Kim Lam¹, Soh Kim Geok², Rosna Abdul Raman' \\ ${ }^{1}$ Nursing Unit, Department of Medicine, Faculty of Medicine and Health Science, Universiti Putra Malaysia, \\ Malaysia \\ ${ }^{2}$ Department of Sport Studies, Faculty of Education, Universiti Putra Malaysia, Malaysia \\ Email: ${ }^{\text {j_salimah@upm.edu.my }}$
}

Received December 2014

\begin{abstract}
This study was carried out to determine the relationship between ethnicity/culture and analgesic consumption among patients from three main ethnic groups in Malaysia after breast cancer surgery. A retrospective study design was adopted, using medical records of 80 patients. The findings showed that the analgesic most consumed by the patients during the 48 hour post-operative period was morphine equivalents $(85 \%)$, followed by non steroidal anti-inflammatory drugs (60\%) and paracetamol (38\%). No relationship was found between ethnicity/culture and the type or amount of analgesic consumption.
\end{abstract}

Keywords

Analgesic Consumption, Breast Cancer Surgery, Culture, Ethnicity, Malaysia

\section{Introduction}

Past studies on culture and pain have revealed disparities in pain management practices, particularly concerning analgesic prescriptions among minority groups [1]-[3]. Several factors may influence these practices, including patients' attitudes, language barriers, heterogeneity of groups, and the medical costs involved [4]. A retrospective cohort study conducted in a hospital emergency department in Atlanta disclosed that only $57 \%$ of African American patients received adequate analgesia compared to $74 \%$ of White American patients $(p=0.01)$ [3]. This study also found that even after controlling variables such as insurance status and total time in the emergency department, the African Americans remained identifiable as a group that received less analgesia in the emergency department [3].

Another retrospective study was carried out among post-abdominal surgical patients in Australia to determine

${ }^{*}$ Corresponding author.

How to cite this paper: Japar, S., Lam, S.K., Geok, S.K. and Raman, R.A. (2015) The Influence of Culture on Analgesic Consumption: A Retrospective Study among Malaysian Patients after Breast Cancer Surgery. Journal of Biosciences and Medicines, 3, 12-17. http://dx.doi.org/10.4236/jbm.2015.33003 
the influence of patients' demographic characteristics, including ethnicity, on the prescription of patient-controlled analgesia (PCA) or intra-muscular analgesia for post-operative pain [2]. The number of patients from ethnic minorities (Lebanese, Italians, Vietnamese and Chinese) who received PCA was found to be lower than for Australian-born patients, thus indicating that ethnic origin had a significant influence on PCA prescriptions $(p=0.034)$. It was suggested that the difference in pain assessment and physician decision-making regarding the prescription and administration of PCA to these groups of patients might have been due to communication barriers between these patients and their physicians [2]. However, this study did not report any data regarding patients' pain intensity levels which might account for the differences in PCA prescription and administration. The retrospective designs and analyses of the secondary data used in both studies discussed above might suffer from recall bias as some data extracted from the existing reports were not well documented [2] [3]. Moreover, data from secondary reports might be incomplete or inaccurate. Finally, in a study by Todd et al. [3], no pain levels were measured and used to describe the patients' pain condition, which might have influenced analgesic prescriptions.

One particular study showed that ethnicity indeed influenced patients' pain and analgesic prescriptions [4]. This post-operative pain study was conducted among women in Singapore who had undergone a lower uterine segment caesarean section procedure. The results indicated that women from the Indian community, a minority ethnic group in Singapore, received a higher amount of analgesia compared to most Malay and Chinese women. The findings from this study also showed significant differences in pain levels and analgesic consumption among the three ethnic groups. However, as the distribution of ethnic groups was not representative of the general population, the findings cannot be generalised. Furthermore, factors such as psychological and socio-cultural beliefs which might have been confounding factors were not explored. The patient's cultural background affects his or her way of coping with pain. Every culture has its own beliefs, interpretation of pain, and ways of managing pain [5] [6]. Therefore, this study aimed to determine the relationship of analgesic consumption vis-àvis ethnicity and culture among patients from three main ethnic groups in Malaysia, namely Malay, Chinese and Indian, who had undergone breast cancer surgery. Hence, the findings from this study might provide some cultural insights to be used by health care providers as a guide in their care for patients of different ethnic groups.

\section{Materials and Methods}

In a retrospective study, the medical records of 123 patients who underwent breast cancer surgery from February to July 2011 were reviewed. Eighty medical records met the criteria for this study where patients included in the study were Malay, Chinese or Indian (both parents of the same ethnicity). They were aged 18 years and above and were able to read and understand Malay or English. The data collected included the patients' demographic details (ethnic group, religion, educational level and age), type of surgery and the type and amount of analgesic used. The patient's pain level was assessed three times (4, 24 and 48 hours) after operation using a visual analogue scale (VAS). The VAS consisted of a $100 \mathrm{~mm}$ line that had the descriptor "no pain" at one end and "worst pain possible" at the other. The patient marked on the line the point that represented her level of pain. Data on analgesic consumption was recorded on the occasions the pain levels were assessed. The patients stayed in hospital for at least 2 days after operation. Patients who had cognitive disorder or were under palliative care were excluded from this study. Data collection was conducted in two government hospitals which are major tertiary referral hospital for breast cancer sufferers in Malaysia.

\section{Results}

The majority of patients who participated in this study were ethnic Malays $(n=56 ; 70 \%)$ followed by Chinese $(\mathrm{n}=15 ; 19 \%)$ and Indian $(\mathrm{n}=9 ; 11 \%)$. Among the patients, the predominant religion was Islam $(\mathrm{n}=57 ; 71 \%)$ followed by Buddhism ( $n=12 ; 15 \%)$, Hinduism $(n=8 ; 10 \%)$ and Christianity $(n=3 ; 4 \%)$. The age of the patients ranged from 19 to 72 years, with a mean age of 53.0 years $(S D=11.2$ years). Upper secondary schooling was the highest level of education reached by the majority of the patients $(n=67 ; 84 \%)$. The majority of patients had undergone surgery for the first time ( $\mathrm{n}=74 ; 93 \%)$, with the most common surgical type for the patients in this study being "mastectomy and axillary node dissection" ( $n=60 ; 75 \%)$.

Pain scores were assessed at 4, 24, and 48 hours post-operation (Figure 1). During these three post-operative periods, more than $50 \%$ of the patients in all ethnic groups experienced pain scores of $50 \mathrm{~mm}$ and less. The number of patients who experienced pain that elicited scores of more than $50 \mathrm{~mm}$ decreased with time. 


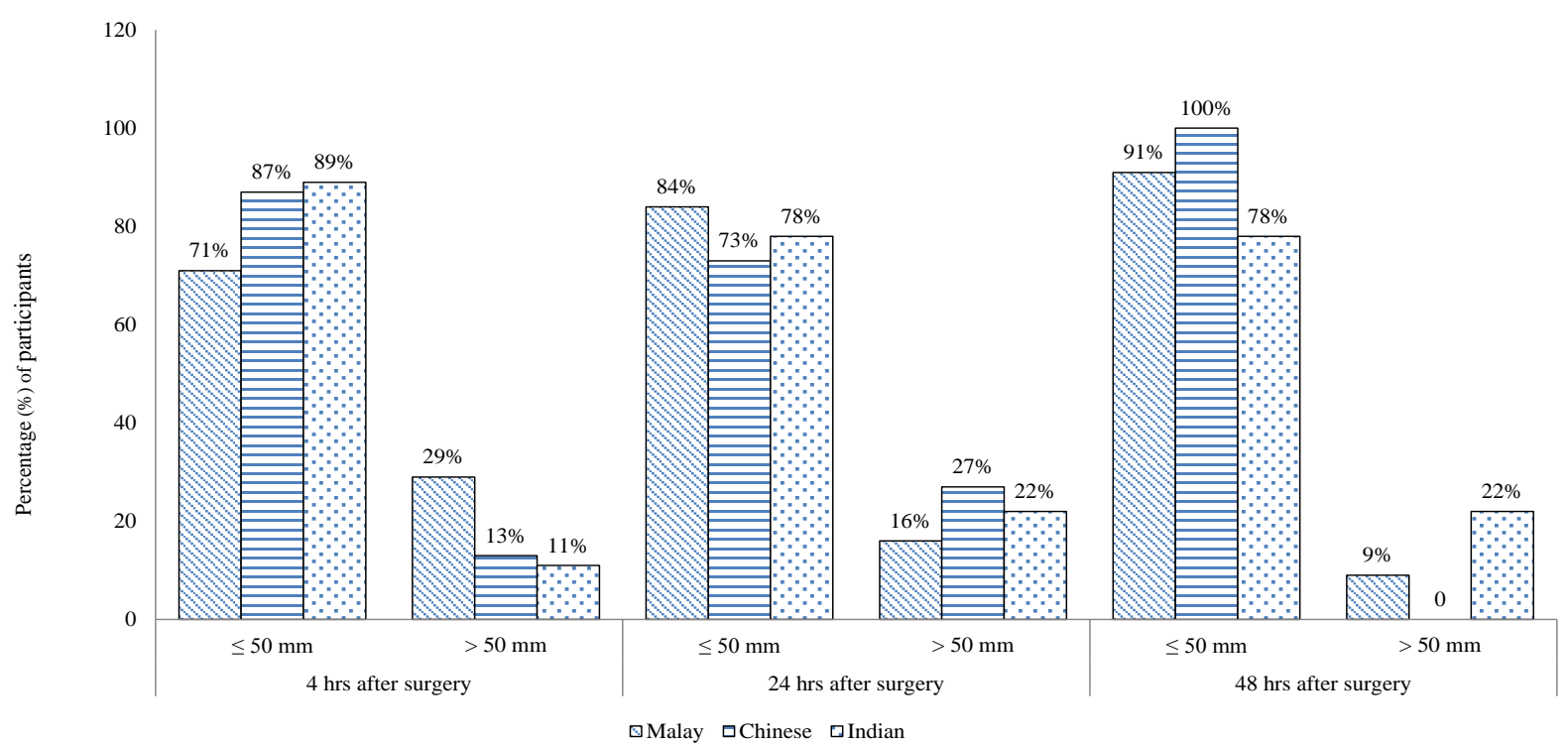

Figure 1. Pain level scores $(\mathrm{mm})$ at 4,24 , and 48 hours after surgery $(\mathrm{n}=80)$.

The types of analgesic prescribed to participants were divided into three main categories, namely paracetamol, nonsteroidal anti-inflammatory drugs (NSAIDs), and morphine equivalents. The types of NSAIDs included oral celecoxib, oral diclofenac, and oral mefenamic acid. Morphine equivalents included patient-controlled analgesia (PCA) morphine, intravenous tramadol and oral tramadol, as well as intramuscular injection of pethidine. Results from the analysis (Table 1) showed that more than $80 \%$ of the participants $(n=68)$ consumed the analgesic type of morphine equivalents (morphine, tramadol or pethidine) throughout the 48 hour postoperative period. Some participants also consumed more than one type of analgesic at one time. Cramer's $V$ test showed no significant relationship between ethnicity and type of analgesic (paracetamol, NSAIDs and morphine equivalents) (Table 1) consumed.

The total dosage for each type of analgesic was divided at the median cut-off point for the whole sample since the data were non-normally distributed. Table 2 presents the total dosage of analgesic consumption by participants across the ethnic groups during the 4 hour, 24 hour and 48 hour postoperative periods.

At the first four hours after their operation, only 17 (21\%) participants had taken some form of analgesic, while 63 (79\%) participants did not. Fifteen participants (19\%) were prescribed morphine equivalents, with the majority of them having a morphine dosage of $10 \mathrm{mg}$ or lower $(\mathrm{n}=12 ; 15 \%)$. Only one participant (Indian) consumed both NSAIDs and paracetamol, with a dosage of $1000 \mathrm{mg}$ and $200 \mathrm{mg}$ respectively. The total analgesic consumption increased across all types of analgesics during the 24 hour post-operative period as compared to the first 4 hours. In the 48 hour postoperative period, the number of participants and the total dosage of morphine equivalents remained similar to the amounts consumed during the 24 hour postoperative period. The results showed no significant relationship between ethnicity and amount of analgesic consumed during the three postoperative time periods (Table 2). The relationship between ethnicity and total paracetamol and NSAIDs dosage could not be established in the 4 hour postoperative period as only one participant used paracetamol or NSAIDs during this period.

\section{Discussion}

In this study, morphine equivalents (morphine, pethidine, and tramadol) were predominantly consumed by participants, followed by NSAIDs (celecoxib, diclofenac, mefenamic acid) and paracetamol. Most participants in the present study consumed more than one analgesic at one time, through a combination of morphine equivalents and NSAIDs or paracetamol. The analgesic prescriptions in both hospitals were based on the 2004 National Protocol of Pain Management [7]. For the patients in the present study, morphine equivalents were prescribed for moderate to severe pain, often given immediately after their operation. Paracetamol and NSAIDs were prescribed for mild to moderate pain [7]. This practice of combination therapy was consistent with an earlier study 
Table 1. Relationship between ethnicity and type of analgesic $(n=80)$.

\begin{tabular}{ccccc}
\hline \multirow{2}{*}{ Type of analgesic } & \multicolumn{3}{c}{ Ethnicity } & \multirow{2}{*}{$p$ value } \\
\cline { 2 - 4 } & Malay $(\mathrm{n}=56) \mathrm{n}(\%)$ & Chinese $(\mathrm{n}=15) \mathrm{n}(\%)$ & Indian $(\mathrm{n}=9) \mathrm{n}(\%)$ & \\
\hline Paracetamol & $17(30)$ & $8(53)$ & $5(56)$ & Cramer's $V=0.226, p=0.130$ \\
NSAIDs & $36(64)$ & $8(53)$ & $4(44)$ & Cramer's $V=0.176, p=0.290$ \\
Morphine equivalents & $47(84)$ & $14(93)$ & $7(77)$ & Cramer's $V=0.124, p=0.539$ \\
\hline
\end{tabular}

Table 2. Relationship between ethnicity and total dosage of analgesic $(n=80)$.

\begin{tabular}{|c|c|c|c|c|c|c|}
\hline \multirow{2}{*}{$\begin{array}{c}\text { Type of } \\
\text { analgesic at } \\
\text { post-operative } \\
\text { points }\end{array}$} & \multirow[b]{2}{*}{ Dosage } & \multicolumn{4}{|c|}{ Ethnicity } & \multirow[b]{2}{*}{$p$ value } \\
\hline & & $\begin{array}{l}\text { Total } \\
\text { n (\%) }\end{array}$ & $\begin{array}{c}\text { Malay }(\mathrm{n}=56) \\
\mathrm{n}(\%)\end{array}$ & $\begin{array}{c}\text { Chinese }(\mathrm{n}=15) \\
\mathrm{n}(\%)\end{array}$ & $\begin{array}{c}\text { Indian }(\mathrm{n}=9) \\
\mathrm{n}(\%)\end{array}$ & \\
\hline \multicolumn{7}{|l|}{4 hours } \\
\hline Paracetamol & $\leq 1000 \mathrm{mg}$ & $1(1)$ & $0(0)$ & $0(0)$ & $1(11)$ & - \\
\hline \multirow{3}{*}{ NSAID } & $>1000 \mathrm{mg}$ & $0(0)$ & $0(0)$ & $0(0)$ & $0(0)$ & \\
\hline & $\leq 200 \mathrm{mg}$ & $1(11)$ & $0(0)$ & $0(0)$ & $1(11)$ & - \\
\hline & $>200$ mg & $0(0)$ & $0(0)$ & $0(0)$ & $0(0)$ & \multirow[b]{2}{*}{ Cramer's $V=0.35, p 0.392$} \\
\hline $\begin{array}{l}\text { Morphine } \\
\text { equivalent }\end{array}$ & $\begin{array}{l}\leq 10 \mathrm{mg} \\
>10 \mathrm{mg}\end{array}$ & $\begin{array}{c}12(15) \\
3(4)\end{array}$ & $\begin{array}{l}7(13) \\
3(5)\end{array}$ & $\begin{array}{c}4(27) \\
0(0)\end{array}$ & $\begin{array}{c}1(11) \\
0(0)\end{array}$ & \\
\hline \multicolumn{7}{|l|}{24 hours } \\
\hline Paracetamol & $\leq 2000 \mathrm{mg}$ & $14(18)$ & $6(11)$ & $5(33)$ & $3(33)$ & \multirow{2}{*}{ Cramer's $V=0.270, p 0.418$} \\
\hline \multirow{3}{*}{ NSAID } & $>2000 \mathrm{mg}$ & $10(13)$ & $7(13)$ & $2(13)$ & $1(11)$ & \\
\hline & $\leq 200 \mathrm{mg}$ & $22(28)$ & $15(27)$ & $4(27)$ & $3(33)$ & \multirow{2}{*}{ Cramer's $V=0.199, p 0.490$} \\
\hline & $>200 \mathrm{mg}$ & $14(18)$ & $12(21)$ & $1(7)$ & $1(11)$ & \\
\hline $\begin{array}{l}\text { Morphine } \\
\text { equivalent }\end{array}$ & $\begin{array}{l}\leq 20 \mathrm{mg} \\
>20 \mathrm{mg}\end{array}$ & $\begin{array}{l}45(56) \\
22(28)\end{array}$ & $\begin{array}{l}31(55) \\
15(27)\end{array}$ & $\begin{array}{l}8(53) \\
6(40)\end{array}$ & $\begin{array}{l}6(67) \\
1(11)\end{array}$ & Cramer's $V=0.161, p 0.421$ \\
\hline \multicolumn{7}{|l|}{48 hours } \\
\hline Paracetamol & $\leq 5500 \mathrm{mg}$ & $15(19)$ & $8(14)$ & $4(27)$ & $3(33)$ & \multirow{2}{*}{ Cramer's $V=0.093, p 0.879$} \\
\hline \multirow{3}{*}{ NSAIDs } & $>5500 \mathrm{mg}$ & $15(19)$ & $9(16)$ & $4(27)$ & $2(22)$ & \\
\hline & $\leq 600 \mathrm{mg}$ & $35(44)$ & $25(45)$ & $7(47)$ & $3(33)$ & \multirow{2}{*}{ Cramer's $V=0.163, p 0.520$} \\
\hline & $>600 \mathrm{mg}$ & $14(18)$ & $12(80)$ & $1(7)$ & $1(11)$ & \\
\hline $\begin{array}{l}\text { Morphine } \\
\text { equivalent }\end{array}$ & $\begin{array}{l}\leq 20 \mathrm{mg} \\
>20 \mathrm{mg}\end{array}$ & $\begin{array}{l}36(45) \\
32(40)\end{array}$ & $\begin{array}{l}25(45) \\
22(39)\end{array}$ & $\begin{array}{l}7(47) \\
7(47)\end{array}$ & $\begin{array}{l}4(36) \\
3(27)\end{array}$ & Cramer's $V=0.038, p 0.952$ \\
\hline
\end{tabular}

in a cohort of postoperative patients with breast surgery where it was found that NSAIDs with opioids or paracetamol reduced opioid consumption during the first six hours and yet provided improved pain relief [8]. Paracetamol in combination with NSAIDs resulted in better and longer pain relief and reduced the side-effects of NSAIDs [7].

This study found that ethnicity and culture did not significantly influence the type or amount of analgesia doctors prescribed to cope with the level of pain experienced by their patients. While it appears there has been limited study examining the relationship between culture and analgesic consumption among breast cancer patients specifically in Malaysia, this relationship has been explored in other cohorts. Earlier studies among patients in the United Kingdom and Singapore examined the issue from the perspective of differences between ethnic groups and analgesia. The study, conducted in the United Kingdom among patients in an emergency unit, 
demonstrated a similar result where there was no significant difference between culture and analgesic use among Bangladeshi and Caucasian patients [9]. In contrast, a Singaporean study by Tan et al. [10] reported a significant difference between culture and morphine consumption among Malay, Chinese and Indian patients, with Indian patients consuming the most. Although the ethnic groups involved in the study by Tan et al. were similar to those of the present study, the different findings might be due to the fact that the participants in the Singapore study underwent a different type of surgery, i.e. caesarean operation, and so consumed a different type and quantity of analgesia. Furthermore, as the sample size in the study by Tan et al. was far larger than that in the present study, which about 1034 participants involved, therefore it was an increased likelihood of detecting a statistical relationship between ethnicity and pain as well as ethnicity and analgesic consumption.

The present study revealed that many participants experienced untreated pain. Based on the participants' medication records, approximately $79 \%$ of participants did not receive any analgesic during the first four hours after their operation. During this time, about $24 \%$ of the participants reporting pain at a level exceeding a score of $50 \mathrm{~mm}$ on the pain level scale. All the participants had been prescribed with analgesic, but the analgesic was not always administered by nurses during the 4 hour postoperative period. Untreated acute postoperative pain is likely to hamper recovery from surgery, and if patients remain in severe pain this can lead to chronic pain such as post-mastectomy pain syndrome [11]. Past studies have revealed several factors that might explain why many patients in this study did not receive any analgesic during the first four hours in the postoperative period. For instance, lack of analgesic availability at the time of patients' need and shortage of nurses are the reasons why patients do not receive the medication on time [12]. Other reasons might include the set routines of analgesic administration in the ward, and patients' attitudes.

A study by Kalisch and Tschannen et al. [12] conducted in an acute care setting in 10 hospitals in the United States found that failure to administer medication on time was among the top five care elements overlooked by nurses. In Malaysian government hospitals generally, only analgesics such as morphine, pethidine, diclofenac, and paracetamol are available in the ward at any time. Other analgesics (opioid or NSAIDs) such as tramadol and celecoxib that have been prescribed by the doctor must be ordered from the pharmacy. Waiting for the analgesics to arrive in the ward sometimes delays the administration of the medication to the patient. Thus, patients might not receive the analgesic on time.

A shortage of nurses also may be related to increasing workload [13], thus resulting in patients not receiving analgesics on time. In the researcher's experience, in order to lighten the workload, some nurses follow a fixed schedule for the administration of medication. For instance, the analgesic intravenous tramadol prescribed eight hourly (three times per day) would be administered at 08:00, 16:00 and 23:00. In the present study, many patients did not receive the analgesic during the first four hour post-operative period, and this was most probably due to that time period not coinciding with the scheduled time for analgesic administration. In addition, the reason that some patients did not receive analgesic in the 4 hour post-operative period might be that many patients had received intra-operative analgesia; therefore nurses were reluctant to administer analgesic early in the postoperative period. Moreover, the nurse-to-patient ratios in both hospitals in the present study were approximately 1:8 to 1:10, which may suggest pressure on the nurses' workload. In future research, the relationship between nurses' workload and medication administration should be further explored.

Some studies have found that patients' attitudes, such as reluctance or refusal to have an analgesic, desire to be a good patient [14] [15] and fear of side-effects of analgesics [16] [17], are related to untreated postoperative pain, and these factors may possibly explain why few analgesics are administered in the 4-hour postoperative period. A Taiwanese study among 207 patients who had undergone several types of surgery observed that 21\% of patients were reluctant to report post-operative pain during the first three days after their operation, and 33\% refused to use analgesics during this period. The findings also revealed a significant relationship between patients who were reluctant to report pain and their reluctance to use analgesics for post-operative pain, compared to patients who reported the pain. In addition, a significant relationship was found between reluctance to use analgesics and the desire to be a good patient [14] [15].

\section{Conclusion}

In conclusion, this study found no relationship between ethnicity/culture, and type of analgesic as well as the amount of analgesic consumed among female patients from three main ethnic groups in Malaysia who underwent breast cancer surgery. However, the study also found it likely that analgesic was not administered in a 
timely manner to the majority of the patients in the 4, 24 and 48 hour postoperative periods. Further studies could investigate factors for the non-administration of analgesic as scheduled or as needed by patients.

\section{Acknowledgements}

The authors would like to express special thanks to the Director General of Health Malaysia for permission to publish this paper, and to hospital directors for their cooperation in this study.

\section{References}

[1] Lee, E., Teeple, M., Bagrodia, N., Hannallah, J., Yazzie, N.P. and Adamas-Rappaport, W.J. (2013) Postoperative Pain Assessment and Analgesic Administration in Native American Patients Undergoing Laparoscopic Cholecystectomy. JAMA Surgery, 148, 91-93. http://dx.doi.org/10.1001/jamasurg.2013.682

[2] Salamonson, Y. and Everett, B. (2005) Demographic Disparities in the Prescription of Patient-Controlled Analgesia for Postoperative Pain. Acute Pain, 7, 21-26. http://dx.doi.org/10.1016/j.acpain.2004.11.001

[3] Todd, K.H., Deaton, C., D’Adamo, A.P. and Goe, L. (2000) Ethnicity and Analgesic Practice. Annals of Emergency Medicine, 35, 11-16. http://dx.doi.org/10.1016/S0196-0644(00)70099-0

[4] Ezenwa, M.O., Ameringer, S., Ward, S.E. and Serlin, R.C. (2006) Racial and Ethnic Disparities in Pain Management in United States. Journal of Nursing Scholarship, 38, 225-233. http://dx.doi.org/10.1111/j.1547-5069.2006.00107.x

[5] Davidhizar, R. and Giger, J.N. (2004) A Review of the Literature on Care of Clients in Pain Who Are Culturally Diverse. International Nursing Review, 51, 47-55. http://dx.doi.org/10.1111/j.1466-7657.2003.00208.x

[6] Narayan, M.C. (2010) Culture’s Effects on Pain Assessment and Management. The American Journal of Nursing, 110, 38-47. http://dx.doi.org/10.1097/01.NAJ.0000370157.33223.6d

[7] Cardosa, M.S., Deva, S.R., Othman, A.S. and Hooi, K.T. (2004) Acute Pain Service. In: Cardosa, M.S., Ahmad, A.B., Idris, N.H. and Yin, W.C., Eds., Pain Management Handbook, Pfizer, Kuala Lumpur, 5-49.

[8] Legeby, M., Sandelin, K., Wickman, M. and Olofsson, C. (2005) Analgesic Efficacy of Diclofenac in Combination with Morphine and Paracetamol after Mastectomy and Immediate Breast Reconstruction. Acta Anaesthesiologica Scandinavica, 49, 1360-1366. http://dx.doi.org/10.1111/j.1399-6576.2005.00811.x

[9] Choi, D.M.A., Yate, P., Coasts, T., Kalinda, P. and Paul, E.A. (2000) Ethnicity and Prescription of Analgesia in an Accident and Emergency Department: Cross Sectional Study. British Medical Journal, 320, 980-981. http://dx.doi.org/10.1136/bmj.320.7240.980

[10] Tan, E.C., Lim, Y., Teo, Y.Y., Goh, R. and Law, H.Y. (2008) Ethnic Differences in Pain Perception and Patient-Controlled Analgesia Usage for Postoperative Pain. The Journal of Pain, 9, 849-855. http://dx.doi.org/10.1016/j.jpain.2008.04.004

[11] Poleshuck, E.L. and Green, C.R. (2008) Socioeconomic Disadvantage and Pain. Pain, 136, 235-238. http://dx.doi.org/10.1016/j.pain.2008.04.003

[12] Kalisch, B.J., Tschannen, D., Lee, H. and Friese, C.R. (2011) Hospital Variation in Missed Nursing Care. American Journal of Medical Quality, 26, 291-299. http://dx.doi.org/10.1177/1062860610395929

[13] Aiken, L.H., Sermeus, W., Van den Heede, K., Sloane, D.M., Busse, R., McKee, M., Bruyneel, L., Rafferty, A.M., Griffiths, P., Moreno-Casbas, M.T., Tishelman, C., Scott, A., Brzostek, T., Kinnunen, J., Schwendimann, R., Heinen, M., Zikos, D., Sjetne, I.S., Smith, H.L. and Kutney-Lee, A. (2012) Patient Safety, Satisfaction, and Quality of Hospital Care: Cross Sectional Surveys of Nurses and Patients in 12 Countries in Europe and the United States. British Medical Journal, 344, e1717.

[14] Soh, K.L., Soh, K.G., Zaiton, A., Rosna, A.R. and Salimah, J. (2008) Perceptions of Stressors in Intensive Care Units among Patients in the Federal Territory Hospitals. Contemporary Nurse, 31, 86-93. http://dx.doi.org/10.5172/conu.673.31.1.86

[15] Tzeng, J.I., Chou, L.F. and Lin, C.C. (2006) Concern about Reporting Pain and Using Analgesics among Taiwanese Postoperative Patients. The Journal of Pain, 7, 860-866. http://dx.doi.org/10.1016/j.jpain.2006.04.007

[16] Cohen, E., Botti, M., Hanna, B., Leach, S., Boyd, S. and Robbins, J. (2008) Pain Beliefs and Pain Management on Oncology Patients. Cancer Nursing, 31, E1-E8. http://dx.doi.org/10.1097/01.NCC.0000305693.67131.7d

[17] Manias, E. and Williams, A. (2008) Managing Pain in Chronic Kidney Disease: Patient Participation in DecisionMaking. Journal of Advanced Nursing, 61, 201-210. http://dx.doi.org/10.1111/j.1365-2648.2007.04488.x 